\title{
New Method for Forest Resource Data Collection Based on Smartphone Fusion with Multiple Sensors
}

\author{
Guangpeng Fan, ${ }^{1,2}$ Yanqi Dong, ${ }^{1,2}$ Danyu Chen, ${ }^{1,2}$ and Feixiang Chen $\mathbb{D}^{1,2}$ \\ ${ }^{1}$ School of Information Science and Technology, Beijing Forestry University, Beijing 100083, China \\ ${ }^{2}$ Engineering Research Center for Forestry-oriented Intelligent Information Processing, \\ National Forestry and Grassland Administration, Beijing 100083, China \\ Correspondence should be addressed to Feixiang Chen; bjfxchen@bjfu.edu.cn
}

Received 4 February 2020; Revised 19 April 2020; Accepted 6 May 2020; Published 18 May 2020

Academic Editor: Sungchang Lee

Copyright (c) 2020 Guangpeng Fan et al. This is an open access article distributed under the Creative Commons Attribution License, which permits unrestricted use, distribution, and reproduction in any medium, provided the original work is properly cited.

\begin{abstract}
Tree parameter measurement is an important part of forest resource monitoring. Smartphones play an important role in forest resource surveys. Although sensors inside smartphones, such as gyroscopes and angle sensors, can meet the needs of the public for entertainment or games, the measurement accuracy in professional forest resource monitoring is slightly insufficient. In this paper, a method of collecting tree measurement factors based on personal smart space fusion with a variety of high-precision sensors is proposed. First of all, a high-precision attitude sensor measurement module and a laser ranging module are organically integrated and packaged in a black box. The smartphone is then connected to the sensor box using a magnet sheet, and the working personnel can determine key parameters in the forest stand by holding it. Finally, in order to verify the accuracy of the method, the measured values in this paper are compared with the reference values. The root mean square error (RMSE) of the tree position in the $X$ and $Y$ directions was $0.114 \mathrm{~m}$ and $0.147 \mathrm{~m}$, the relative deviations (rBias) were $0.95 \%$ and $0.39 \%$, and the average RMSE was $0.186 \mathrm{~m}$. The RMSEs measured by tree height and diameter at breast height (DBH) were $0.98 \mathrm{~m}$ and $2.24 \mathrm{~cm}$, the relative root mean square error (rRMSE) was $5.87 \%$ and $13.46 \%$, and the relative deviations (rBias) were $-1.40 \%$ and $-1.06 \%$, respectively. Therefore, the method of forest stand parameter measurement based on personal smart space fusion multitype sensors proposed in this paper can be effectively applied to forest resource data collection.
\end{abstract}

\section{Introduction}

Forests play an important role in maintaining ecosystem balance, protecting the environment, responding to climate change, sequestering carbon, maintaining soil and water, and providing forest products [1-3]. Mastering and understanding detailed forest information can help us strengthen forest protection. Some researchers took advantage of smartphones with various sensors such as angle sensors, orientation sensors, and gyroscopes and applied smartphones to forest resource monitoring $[4,5]$. Some researchers proposed to measure key tree measurement factors such as tree species, diameter at breast height (DBH), tree height, and tree position based on smartphones and store the measured tree measurement factors in the database of the smartphone $[6,7]$. Some researchers developed tree height measurement software running on smartphones using the principle of trigonometric functions. These studies can theoretically measure accurate geometric lengths, but many smartphones have large deviations in sensor accuracy such as angle sensors and direction sensors [8-10]. In general, smartphones can meet the accuracy requirements of mass entertainment or game consumption. However, when applied to professional fields such as forest resource monitoring, the sensitivity of the sensors built in the smartphones will cause unstable measurement errors [11]. Some researchers have used the Global Navigation Satellite System (GNSS) function of smartphones in forest stand positioning, but such methods have significant limitations and are greatly affected by stand density [12-14]. Especially in some forest 
areas where the GNSS signal is weak or there is no signal, the positioning accuracy of the smartphone is low or the positioning function cannot be used. Some researchers combined smartphones, laser rangefinders, and tripods to work in the forest [15]. Although this method can meet the functional requirements of forest resource survey, it can also lead to the problem of poor portability in practical work, especially in the face of hillside fields, which will affect the observation of workers.

At present, UAV, light detection and ranging (LiDAR), remote sensing, and photogrammetry have gradually become important technologies for forest resource monitoring [16-18]. A forest resource monitoring system integrating sky and ground and air has been formed and is applicable to different work scenarios [19]. Among the existing forest ground monitoring methods, in addition to the traditional tree measuring instruments, terrestrial photogrammetry, ground-based LiDAR, and other technologies have also emerged, which can not only obtain accurate tree location, tree height, $\mathrm{DBH}$, and other basic tree measurement factors but also promote the computerization of forestry [20-22]. LiDAR technology also has some problems in forest resource monitoring, such as complicated operation, high investigation cost, poor portability, and large computation [23]. Photogrammetry is relatively cheap compared with LiDAR, but photogrammetry requires multiple independent digital cameras, and the measurement results need to be returned to the office for postprocessing [24], which cannot be solved in real time on work field. Therefore, LiDAR technology has many limitations in small-scale or low-cost forest surveys [25]. The tree position is necessary to accurately calculate and measure the characteristics of the forest and describe the changes in the forest [26]. Especially in the work of establishing a fixed plot, it is necessary to obtain accurate tree positions $[27,28]$. In the early investigation stage, the relative position of the trees was mainly determined by using the compass to obtain the azimuth and the rope to obtain the horizontal distance [29]. With the application of GNSS in forestry, GNSS-based stand positioning technology has gradually matured. Therefore, GNSS positioning technology based on smartphones will also produce large measurement errors in dense forest stands [30]. It is necessary to develop a relative positioning method to measure the position of trees based on smartphones. The DBH and tree height of standing trees are important contents of forest resource surveys and are also the basic factors for estimating forest accumulation and biomass [31, 32]. Taking advantage of the relatively low price of photogrammetry, it is necessary to develop a smartphone-based photogrammetry method to obtain the $\mathrm{DBH}$ and height of trees. In the current research on forest resource monitoring based on smartphones, there are problems of unstable measurement errors due to low accuracy of the sensor, as well as problems of single function or poor portability $[4,8,33]$.

In the measurement of tree attributes, we need to obtain the key parameters of the standing tree (including factors such as position, $\mathrm{DBH}$, and tree height). In order to make smartphones more convenient to obtain high-precision azimuth and horizontal distance, this paper proposes a method for measuring tree attributes based on personal smart space fusion with multiple sensors. Considering the existence of hillside fields in forest resource monitoring, from the perspective of portability, a variety of high-precision sensors (including angle sensors, orientation sensors, gyroscopes, and laser ranging modules) are integrated into a small box that is easy to carry, and this paper defines it as "sensor box." The "sensor box" is tightly connected to the back of the smartphone through a magnet sheet, which makes it easier for forest workers to carry around. The purposes of this paper are as follows. (1) In view of the lack of accuracy or sensitivity of the sensors of smartphones in forest resource monitoring, a method of measuring key parameters of forest stands by integrating a variety of highprecision sensors based on smart space is proposed. (2) The position, $\mathrm{DBH}$, and the height of the tree are determined using a relative position positioning algorithm and a standing tree photo processing algorithm. In order to implement the method in this paper, smartphone-based forest stand measurement software was developed to verify the accuracy of the method and the applicability of the method was discussed.

\section{Materials and Methods}

2.1. Sensor Box. In order to improve the problem of low sensitivity of the sensor due to different hardware configurations of the smartphone, this paper has designed a multifunctional sensor called "sensor box" for forest resource monitoring. The sensor box has an attitude measurement function and a distance measurement function. It is mainly used to obtain high-precision 3D data (acceleration, gyroscope, Euler angle, and magnetic field data) and distance measurement data. It can send various types of sensor data to smartphones via Bluetooth. The sensor box overcomes the problem that the accuracy of the sensors built in many smartphones cannot meet the needs of investigation. It differs from the conventional laser rangefinder in the following two aspects. (1) Ordinary laser rangefinders focus on acquiring distance data and cannot acquire gyroscope attitude data. The sensor box designed in this article not only has the function of laser ranging but also has the function of attitude measurement. (2) The ordinary laser rangefinder cannot be physically connected to the smartphone and is considered as a whole. It is necessary for the workers to operate the rangefinder and the smartphone separately. The volume of the sensor box is $80.3^{*} 47^{*} 30 \mathrm{~mm}^{3}$, which is $1 / 2$ of the volume of a conventional laser rangefinder. It can be seamlessly connected to a smartphone through a magnet sheet, and their combination can be considered as a whole.

In this article, a variety of high-precision sensor modules on the market are organically integrated and packaged in a small black box (see Figure 1). The sensor box is mainly composed of an attitude measurement module and a laser ranging module. The attitude measurement module uses the gyro angle measurement module BWT901BLE5.0 provided by WitMotion ShenZhen Co., Ltd. Its $X$-axis and $Y$-axis angle measurement accuracy is $0.01^{\circ}$, and the $Z$-axis angle measurement accuracy is $0.05^{\circ}$. The operating frequency is 


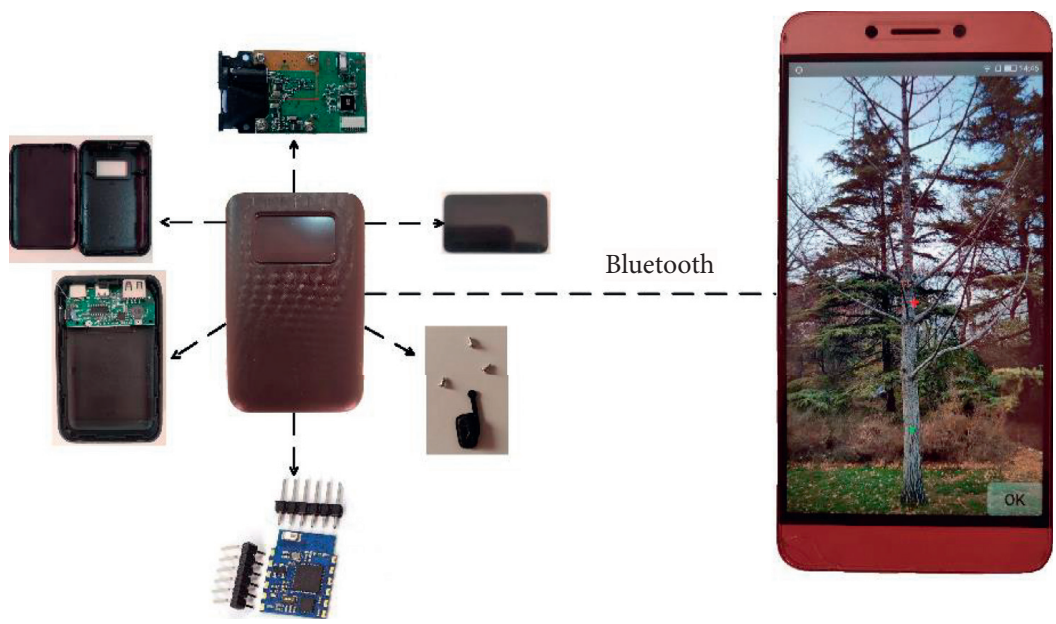

Figure 1: Sensor box and personal smartphone.

$168 \mathrm{MHz}$, the acceleration is $\pm 16 \mathrm{~g}$, the $X$-axis and $Z$-axis angle measurement range is $-180^{\circ} \sim 180^{\circ}$, and the $Y$-axis measurement range is $-90^{\circ} \sim 90^{\circ}$. The baud rate is 2400 921600 bps, and the magnetic field accuracy is $1 \mathrm{mG}$ (milligauss). The return rate is $0.1 \sim 200 \mathrm{~Hz}$. The model of the laser rangefinder module is L4-RS232, which has a measuring range of 0.02-100 meters, a measurement accuracy of $\pm 2 \mathrm{~mm}$, and a laser wavelength and power of 620-690 $\mathrm{nm}$. In Windows 10 operating system, based on Arduino 1.8.5 development environment, $\mathrm{C}++$ programming language is used for serial debugging and secondary development. The sensor box uses a $3.3 \mathrm{v}$ power supply and is equipped with a display to show sensor data.

\subsection{Development of Software for Measuring Tree Attributes} Based on Personal Smart Spaces. This article has developed software that runs on a smartphone to measure the stand. It can accept and process sensor data in the "sensor box" and can calculate the relative position, $\mathrm{DBH}$, and tree height of standing trees. Under Windows 10 operating system, an application development environment based on Android Studio 3.3 + Android SDK (Java Development Kit) + Java JDK 8 (Java Development Kit) + ADT (Android Development Tool) is built. The stand measurement software was written in Java language. The software runs on a smartphone based on the Android operating system (Android 4.4 or higher). The application uses the distance and azimuth provided by the sensor box to calculate the relative position of the trees and uses the gyroscope data to calibrate the smartphone's camera and obtain a fixed focal length. This paper is based on the principle of single image solution and uses the software's "standing tree measurement" module to calculate the tree height and $\mathrm{DBH}$. This article designs a way to connect the sensor box with a smartphone. The measurement personnel can carry out the forest parameter measurement by holding it.

2.3. Research Area. The research in this paper was conducted in an artificially regenerated forest located in
Haidian District $\left(40^{\circ} 0^{\prime} 40^{\prime \prime} \mathrm{N}, 116^{\circ} 20^{\prime} 20^{\prime \prime} \mathrm{E}\right)$, Beijing, China. It is planted with poplar, willow, and locust trees, with little or no undergrowth grass and shrubs. The study area is $48.7 \mathrm{~m}$ above sea level and the terrain is flat. It belongs to the temperate humid monsoon climate zone, with four distinct seasons, hot summers, cold winters, and low precipitation. As shown in Figure 2, this paper has verified the accuracy of the smart space-based method of fusing multiple sensors to measure stands in two temporary sample plots of $40 \times 40 \mathrm{~m}$. The tree density of plantation stands is around 900 trees/ha. To verify the accuracy of the tree location measurement, a total of 67 standing trees were measured. To verify the accuracy of $\mathrm{DBH}$ and tree height measurements, a total of 96 standing trees were measured.

\subsection{Tree Key Parameter Measurement Principle Based on} Smart Space. The sensor box provides a variety of highprecision sensor data for the determination of tree position, $\mathrm{DBH}$, and tree height in this paper. The relative position determination algorithm and standing tree image measurement algorithm are used to process the sensor data collected by the smartphone, and the position, $\mathrm{DBH}$, and height of the standing tree are calculated, respectively. In forest areas with high stand density or severe canopy cover, the GNSS signal strength will be severely affected, resulting in low forest positioning accuracy or inability to meet actual work requirements. Since the GNSS signal of the smartphone into the forest will be severely weakened, it is impossible to directly obtain the absolute position of the trees. Under the guidance of traditional tree position measurement methods [29], this paper provides a new option for forestry investigators to determine the relative position of trees based on smart space fusion with multiple sensors. Based on the principle of close-range photogrammetry $[34,35]$, this paper uses the fixed focal length of a smartphone and the horizontal distance obtained by the sensor box. Then, the height and DBH of the tree can be measured by taking a picture containing the complete information of the standing tree. 

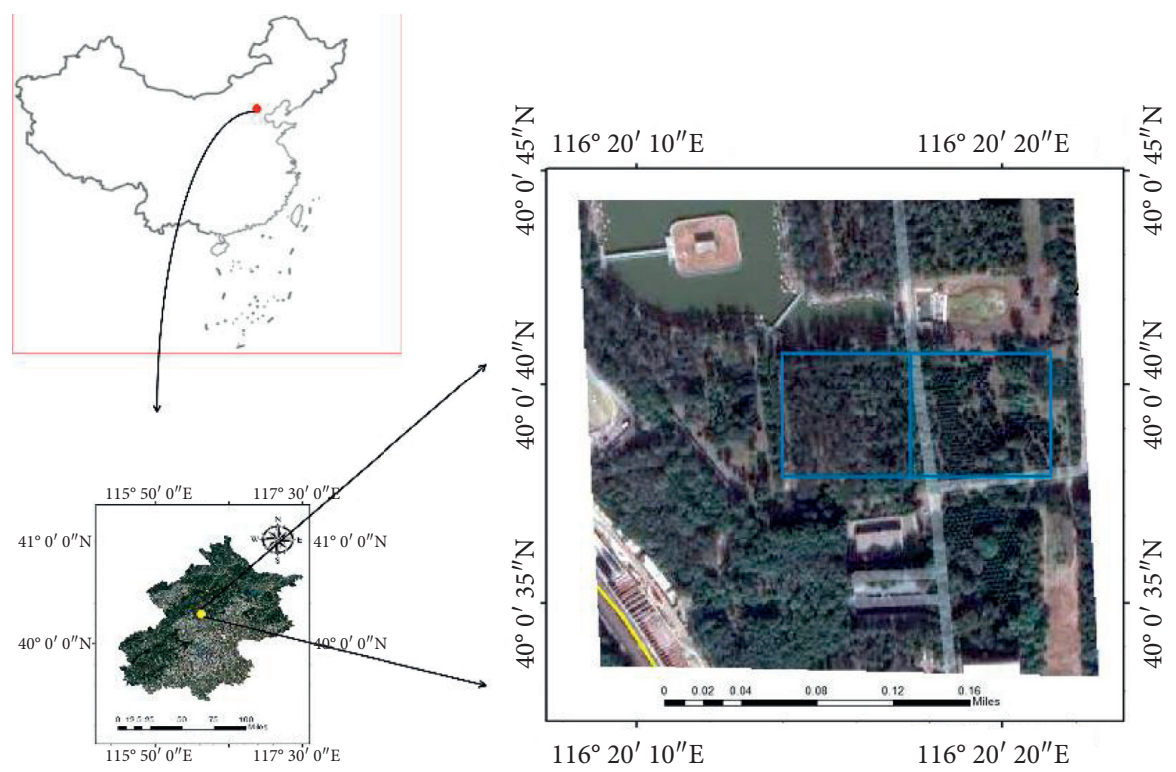

Figure 2: Research area map.

2.4.1. Principle of Standing Tree Position Measurement. First, the surveyor should set the position to the origin (the origin is the initial observation point, whose coordinates are known or assumed), and the location of the origin should be selected as far as possible to the place with wider surrounding field of view. Then, the surveyor opens the "forest stand measurement" software in the smartphone and enters the camera shooting interface of the "standing tree measurement" function and clicks the "Bluetooth" icon in the upper right corner to search and connect the sensor box. According to the actual requirements of the work, if there is a need to measure $\mathrm{DBH}$ and tree height, the laser emitted horizontally by the induction box can be projected to $\mathrm{DBH}$ $(1.3 \mathrm{~m})$ of the trunk by visual judgment. If you just need to measure the position of the tree, you can project the laser horizontally onto the tree trunk anywhere. When the laser point appears in the camera interface, click the "OK" button and keep the arm as stable as possible.

In Figure 3, the tree position coordinate system $O-x y$ is established, and the origin $O$ of the coordinate system is the first observation point. The red triangle represents the observation point, while $O_{x}$ and $O_{y}$ represent the coordinates of the observation point. The green dots represent trees, and $x$ and $y$ represent tree coordinates. $L$ represents the distance from the observation point provided by the calculation box to the tree. $\alpha$ is the azimuth calculated by the induction box. The coordinates of each tree can be calculated using trigonometric functions. For example, the plane coordinates of the first tree are calculated as follows, and the coordinates of other trees are calculated using a similar method.

$$
\left\{\begin{array}{l}
x_{1}=L_{1} * \cos \left(\pi-\alpha_{0}\right), \\
y_{1}=L_{1} * \sin \left(\pi-\alpha_{0}\right) .
\end{array}\right.
$$

When measuring trees at the parcel level, surveyors may encounter situations in which trunks or treetops are blocked due to the density of trees, and some trees are partially visible

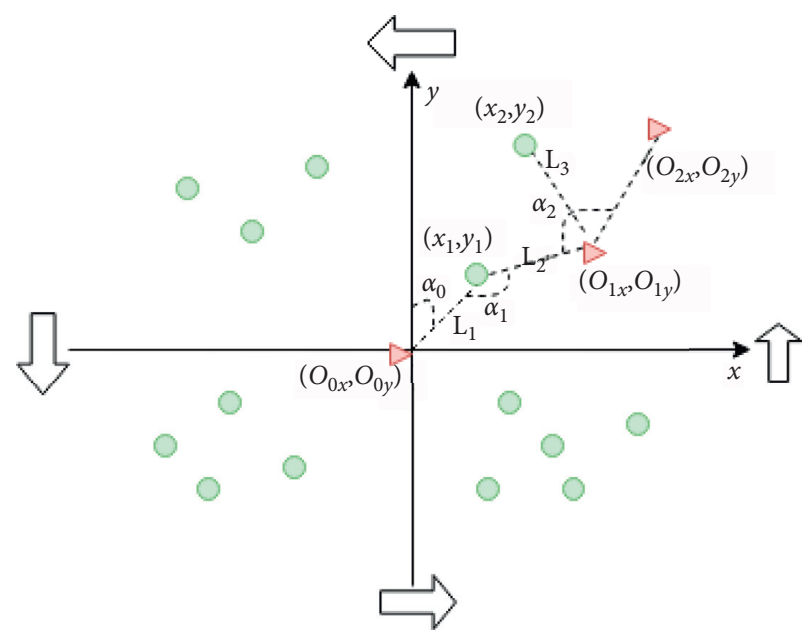

FIgURE 3: Schematic diagram of tree position observation scheme.

or completely invisible. In this paper, an observation mode of "observation point transfer" is designed for surveyors. The measured stands can be divided into two or four plots of similar size according to the density of trees or artificial segmentation markers. The observation principle is to transition from low-density plots to high-density plots. When dividing into two plots, the plot with less dense trees should be observed first. Each plot occupies a quadrant when divided into four cells. Firstly, the plot with the lowest density of trees was taken as the first quadrant, and the observation sequence of anticlockwise or clockwise was determined according to the density of trees in adjacent plots. When the entire stand is divided, the surveyor can start the determination of each stand. If the laser emitted by the sensor box cannot be projected onto the trunk, the surveyor can change the standing position and set up a second observation point. The selection of the second observation point is the same as the first observation point. 
Using the distance data $L$ provided by the sensor box, the coordinates of the second observation point can be inverted according to the coordinates of the previous tree or the coordinates of the measured tree nearby, and then continue the observation. According to the actual needs, the third or fourth observation points are set in the same way.

2.4.2. DBH and Tree Height Measurement of Standing Trees. $\mathrm{DBH}$ and tree height are the key parameters for stand determination. In this paper, the software's "measuring tree" function is used to calculate the chest diameter and tree height of the tree to be measured (see Figure 4). The observation scheme was similar to the observation scheme of tree position. Therefore, this paper realizes the synchronization of measuring the position, $\mathrm{DBH}$, and height of the tree in the actual working process, and the sensor box can provide the data required for calculation at the same time. The tree height and DBH can be calculated based on the method of solving singletall photos. This is a method of single photo in photogrammetry. After entering the "standing tree measurement" module of this software, the surveyor selects the "Image Capture" function. The surveyor will project the laser emitted by the sensor box to the chest diameter $(1.3 \mathrm{~m})$ of the trunk by visual judgment and click the "OK" button. The image of tree will be automatically loaded into the software interface and a red "+" symbol will appear in the center of the smartphone screen. To obtain the exact image plane coordinates of the tree top position, the image of the tree can be moved so that the position $A_{1}$ of the tree is aligned with the "+" symbol, and click the "OK" button in the lower right corner. Then, the image is moved to align the bottom of the tree $A_{2}$ with the "+" symbol. Click the "OK" button to get the image plane coordinates of the point in the image. At this time, the measured value of tree height will appear on the screen. The measurement process of $\mathrm{DBH}$ is similar to that of tree height. The location of DBH is determined by the position of laser point in the photo. Then, by zooming in the image and moving the red "+" symbol, $A_{3}$ to the left of DBH and $A_{4}$ to the right of DBH are determined to complete the acquisition of corresponding coordinates of the image plane. Finally, the DBH measurement value will also appear on the screen.

This algorithm is a special form of collinear equations in photogrammetry and is suitable for calculation of a single image [35-37]. This paper studies the application of this algorithm to smartphones. The measurement principle is shown in Figure 5.

$$
\left[\begin{array}{l}
X \\
Y \\
Z
\end{array}\right]=R\left[\begin{array}{c}
x \\
y \\
-f
\end{array}\right]=\left[\begin{array}{lll}
a_{1} & a_{2} & a_{3} \\
b_{1} & b_{2} & b_{3} \\
c_{1} & c_{2} & c_{3}
\end{array}\right]\left[\begin{array}{c}
x \\
y \\
-f
\end{array}\right],
$$

where $x$ and $y$ are the image plane coordinates of the image points, $f$ is the focal length of the camera of the smartphone, $X$, $Y$, and $Z$ are the object space coordinates of the observation points, and $a_{i}, b_{i}$, and $c_{i}(i=1,2,3)$ are the 9 direction cosines composed of three external azimuth elements of the tree image.

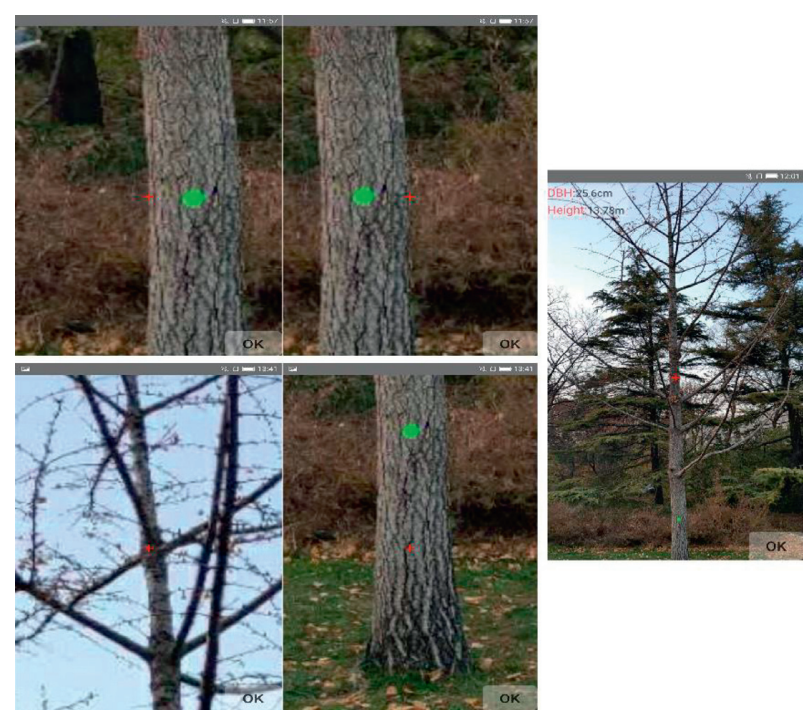

FIGURE 4: Software interface of tree height and DBH measurement.

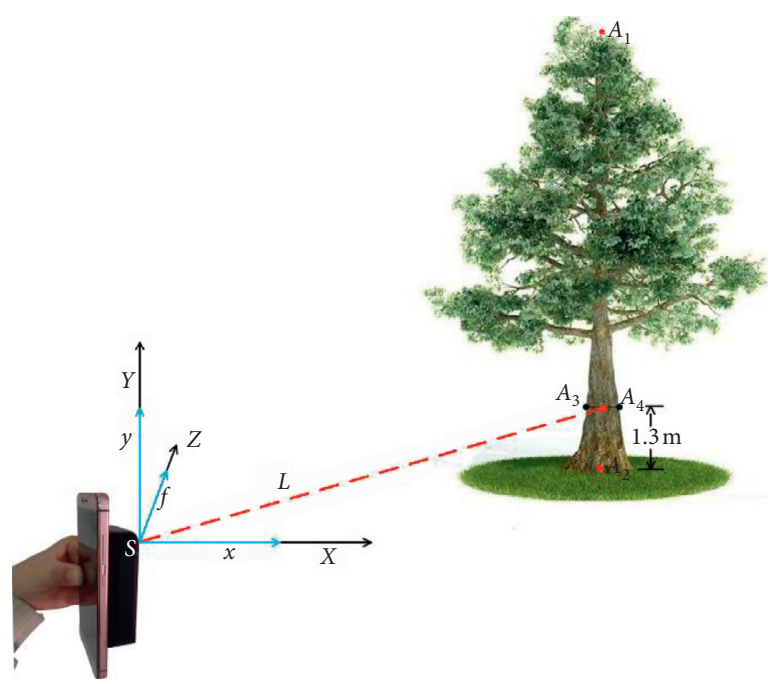

Figure 5: Schematic diagram of standing tree measurement.

In this scenario, the origin of the object space coordinate system and the origin of the image space coordinate system coincide, and the photography center $S$, the image space coordinate point, and the object space coordinate point are on a straight line to simplify the rotation matrix, as shown in the following formula:

$$
\left[\begin{array}{c}
X_{i} \\
Y_{i} \\
Z_{i}
\end{array}\right]=\lambda_{i}\left[\begin{array}{c}
x_{i} \\
y_{i} \\
f
\end{array}\right]
$$

The object space point coordinates of space point $A_{1}$ is set as $\left(X_{A}, Y_{A}, Z_{A}\right)$. Formula (4) can be used to calculate the object-side coordinates of the image points on the standing tree image. 


$$
\left\{\begin{array}{l}
X_{i}=\frac{L x_{i}}{f}, \\
Y_{i}=\frac{L y_{i}}{f}, \\
Z_{i}=L \\
\lambda=\frac{L}{f},
\end{array}\right.
$$

where $\left(X_{i}, Y_{i}, Z_{i}\right)$ is the object space coordinates of the standing tree, $L$ represents the horizontal distance between the observation point and the standing tree, $x_{i}$ and $y_{i}$ are image plane coordinates (2D), and $f$ is the focal length of the camera. The tree height calculation formula is obtained by combining the following formula:

$$
\left\{\begin{array}{l}
\frac{X_{i}}{x_{i}}=\frac{Y_{i}}{y_{i}}=\frac{Z_{i}}{f}=\lambda_{i}, \\
\frac{\sqrt{\left(x_{1}-x_{2}\right)^{2}+\left(y_{1}-y_{2}\right)^{2}}}{\text { height }}=\frac{L}{\mathbf{f}}
\end{array}\right.
$$

and the tree height is

$$
\text { height }=\frac{\sqrt{\left(x_{1}-x_{2}\right)^{2}+\left(y_{1}-y_{2}\right)^{2}} * f}{L},
$$

where $x_{i} y_{i}$ corresponds to the image plane coordinates of $\mathrm{DBH}$ and height of the tree. The tree top $A_{1}$ and the tree bottom $A_{2}$ are selected, respectively, on the image of the tree to be measured. The corresponding image plane coordinates on the image are $\left(x_{1}, y_{1}\right)$ and $\left(x_{2}, y_{2}\right)$, respectively at S. $Z_{i}$ is the scale parameter $(L / f), f$ is the focal length of the smartphone's fixed-focus camera, and $L$ is the horizontal distance between the observation point and the tree to be measured.

Similarly, by selecting the left point $A_{3}$ and right point $A_{4}$ of $\mathrm{DBH}$ on the image, the corresponding image point coordinates (plane coordinates of the image) at $s$ of the image, namely, $\left(x_{3}, y_{3}\right)$ and $\left(x_{4}, y_{4}\right)$, are obtained, respectively. Then, we use the following formulas to calculate the $\mathrm{DBH}$ :

$$
\begin{aligned}
& \left\{\begin{array}{l}
\frac{X_{i}}{u_{i}}=\frac{Y_{i}}{v_{i}}=\frac{Z_{i}}{f}=\lambda_{i}, \\
\frac{\sqrt{\left(x_{3}-x_{4}\right)^{2}+\left(y_{3}-y_{4}\right)^{2}}}{\mathrm{DBH}}=\frac{L}{f},
\end{array}\right. \\
& \mathrm{DBH}=\frac{\sqrt{\left(x_{3}-x_{4}\right)^{2}+\left(y_{3}-y_{4}\right)^{2}} * f}{L} .
\end{aligned}
$$

In the study of measuring standing tree with smartphones, some researchers measured tree based on the principle of trigonometry. In this paper, photogrammetry algorithm was used to measure tree height and $\mathrm{DBH}$. This algorithm requires a known external variable (horizontal distance $L$ between the observation point and the tree) as a "ruler," and its advantages are reflected in the following two points. (1) The trigonometric function tree measurement algorithm needs to measure the observation point and the tree top and bottom, respectively. The angle of the smartphone's angle sensor is relatively low, and the angle error fluctuates greatly. The laser rangefinder only needs to measure the horizontal distance between the observation point and the stand. The accuracy of the distance measurement is very high and the error fluctuation is relatively small. (2) The algorithm in this paper can be used to measure the $\mathrm{DBH}$ and the tree height at the same time by using a smartphone to take a photo containing the complete information of the standing tree. However, the method based on the trigonometric function principle needs to find the tree height before using other algorithms to calculate the $\mathrm{DBH}$.

2.5. Precision Evaluation. In order to verify the validity of this method [38, 39], the measurement accuracy of tree position, $\mathrm{DBH}$, and tree height was analyzed in this paper. In terms of tree position measurement, we take the tree position measured with a total station (KTS-44R4LCN total station, South Surveying \& Mapping Technology Co., Ltd) as a reference value. This paper uses the CGQ-1 direct reading altimeter and $\mathrm{DBH}$ tape used in forestry to measure the height and $\mathrm{DBH}$ of each tree and uses these data as the reference value of the method in this paper. This paper uses Bias, RMSE, rBias, and rRMSE to check the accuracy of tree position, tree height, and $\mathrm{DBH}$.

$$
\begin{aligned}
\text { Bias } & =\frac{1}{n} \sum_{i=1}^{n}\left(y_{i}-y_{r i}\right), \\
\mathrm{RMSE} & =\sqrt{\frac{\sum\left(y_{i}-y_{r i}\right)^{2}}{n},} \\
\mathrm{rBias} \% & =\frac{\operatorname{Bias}}{\bar{y}_{r}} \times 100 \%, \\
\mathrm{rRMSE} \% & =\frac{\mathrm{RMSE}}{\bar{y}_{r}} \times 100 \% .
\end{aligned}
$$

\section{Results}

3.1. Analysis of Tree Position Results. In this paper, 67 trees were measured to verify the accuracy of tree position measurements. During the measurement, the "observation point transfer" scheme in 2.4.1 section was used to solve the problem of trunk occlusion. The total station was used to measure the coordinates of 67 trees and took them as reference values. The position coordinates of trees measured by the fusion of multiple sensors based on personal smart spaces were taken as the measured values.

Figure 6(a) shows a schematic diagram of the distribution of trees under test in the sample plot. It can be clearly seen from Figure 6(b) that the measured value of tree position in this paper can better correspond to the reference 


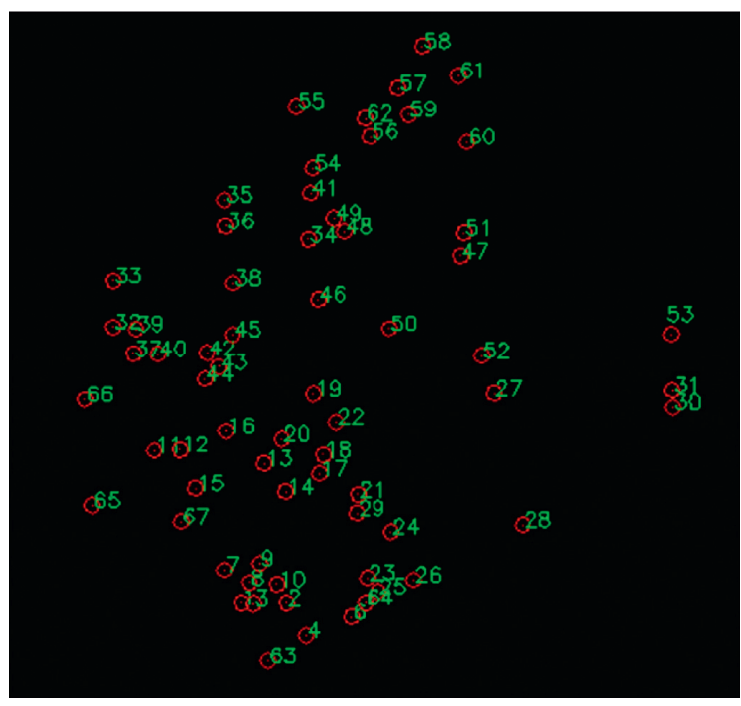

(a)

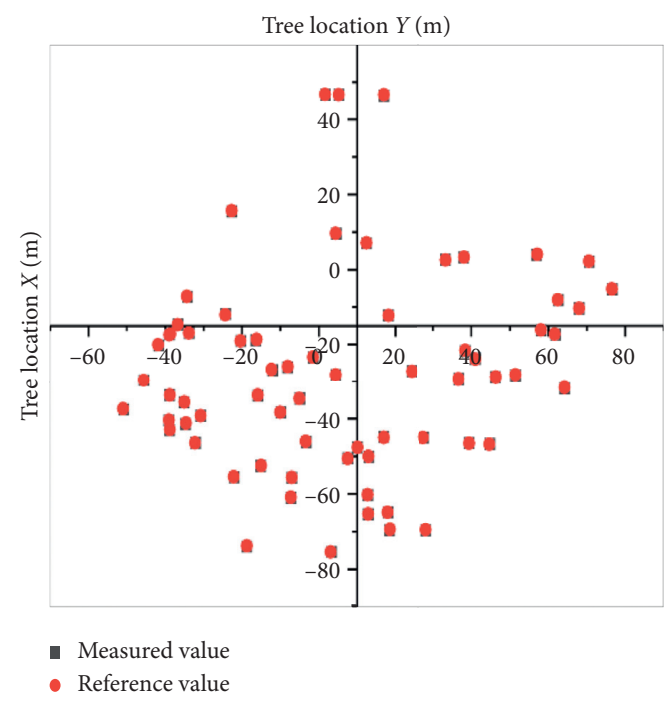

(b)

Figure 6: (a) The distribution of trees in the real world and (b) the comparison of tree position measurements.

value within the scope of sample land. Table 1 shows the Bias, rBias, RMSE, and rRMSE of $X$ and $Y$, respectively.

The average RMSE of tree position in this experiment is $0.186 \mathrm{~m}$, which can better express the distribution of tree position in forest resource monitoring. In practical work, in addition to external factors such as topography and magnetic field, the measurement accuracy of tree position will be affected by the way the surveyors operate. Therefore, the measurement operators should regulate the operation of the instrument, avoid bad measurement environment, and carry out accurate positioning and measurement according to the instructions for the use of the software. The experimental results show that this method can be used to measure the position of trees in forest resource monitoring.

3.2. Analysis of DBH and Height of Standing Tree. In order to verify the accuracy of this method in measuring the $\mathrm{DBH}$ and height of standing trees, 96 living standing trees were measured and these results were taken as measured values. The height and DBH of the standing tree were measured using the CGQ-1 direct reading altimeter and diameter tape, and these results were used as reference values. Figure 7 shows that the measured values of $\mathrm{DBH}$ and tree height are evenly distributed on both sides of the reference value, and the measurement effect is better overall.

The four precision indexes in Table 2 show the measurement results of tree height and $\mathrm{DBH}$. The experimental results show that the stand measurement method based on smart space fusion of multiple sensors can meet the precision requirements of forest resource survey.

In terms of measurement accuracy, although the method in this paper has no obvious advantages compared with existing methods such as LiDAR and photogrammetry, it still provides valuable reference for forest resource monitoring. Therefore, the technology based on smart space fusion of multiple sensors can still be used as an effective
TABLE 1: The comparison of tree position measurement based on relative positioning and total station measurement.

\begin{tabular}{lcccc}
\hline Tree position & Bias & rBias (\%) & RMSE & rRMSE (\%) \\
\hline$X(\mathrm{~m})$ & 0.05 & 0.95 & 0.114 & 2.10 \\
$Y(\mathrm{~m})$ & -0.11 & 0.39 & 0.147 & -0.53 \\
\hline
\end{tabular}

solution for the measurement of tree attributes. The measurement of tree height is directly related to stand density and canopy occlusion. When the stand density is small or the canopy is sparse, the determination accuracy of tree height is high. The accuracy of DBH measurement is mainly affected by picture shooting, finger movement, and other factors. When operating the software, accurately determining the $\mathrm{DBH}$ position is the key to improve the $\mathrm{DBH}$ measurement accuracy.

\section{Discussion}

Intelligent, efficient, and accurate method for measuring tree attributes has always been the focus of forest resource monitoring. Nowadays, with the advantage of multiple sensors, smartphones not only provide a variety of entertainment and consumption-level functions for the public but also have gradually become an important tool in professional fields such as forest resource monitoring $[14,33,39,40]$. However, the sensor accuracy of many smartphones can still meet the entertainment needs, while the accuracy of professional resource detection needs to be improved [39]. In this paper, a high-precision, small sensor box is designed, and it can be seamlessly connected to a smartphone. The sensor box integrates the attitude gyroscope module with the laser rangefinder module to provide high-precision attitude data and horizontal distance data for smartphones. The sensor box has two advantages over the conventional laser rangefinder. (1) The conventional laser rangefinder focuses on the acquisition of distance data but cannot acquire the 


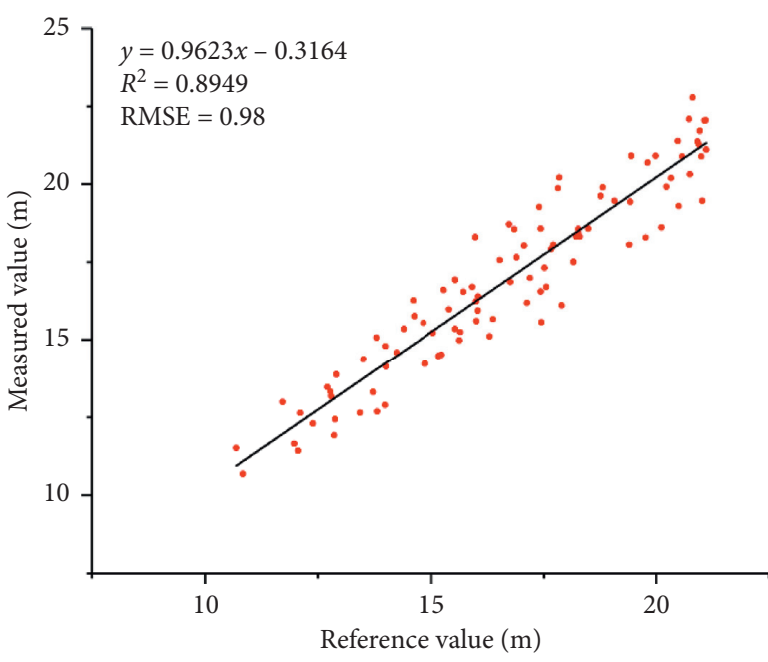

(a)

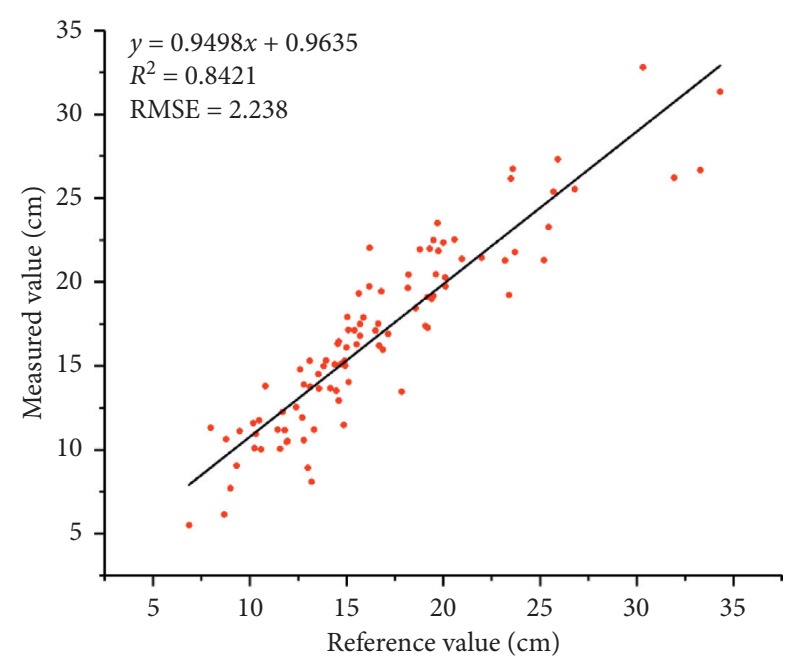

(b)

FIgURE 7: (a) The comparison of tree height measurement and (b) the comparison of DBH measurement.

TABle 2: Comparison of tree measurement based on this method and traditional method.

\begin{tabular}{lcccc}
\hline Category & Bias & rBias (\%) & RMSE & rRMSE (\%) \\
\hline Height $(\mathrm{m})$ & -0.23 & -1.40 & 0.98 & 5.87 \\
DBH $(\mathrm{cm})$ & -0.18 & -1.06 & 2.24 & 13.46 \\
\hline
\end{tabular}

attitude data. The sensor box designed in this paper has not only the function of laser ranging but also the function of attitude measurement. (2) The conventional laser rangefinders cannot be seamlessly connected to smartphones, so surveyors need separate smartphones and laser rangefinders. Such a combination cannot be taken as a whole. At $80.3 * 47 * 30 \mathrm{~mm}$, the sensor box is half the size of a conventional laser rangefinder and can be seamlessly connected to a smartphone via a magnet sheet. Workers can combine the sensor box with the smartphone as a whole. It is easy to carry, easy to operate, and less affected by terrain.

In order to verify the effectiveness of this method, the position, $\mathrm{DBH}$, and height of the tree were compared and analyzed. Although the method presented in this paper has no obvious advantage over LiDAR and photogrammetry in terms of accuracy [17, 23, 24], the measurement method is more flexible and less affected by topographic environment. LiDAR technology is greatly affected by the terrain, it also requires a lot of data processing in the office, and its calculation cost is high. This paper supports foresters to carry out preharvest measurements in the field. A method that combines a sensor box with a smartphone to measure tree properties can be used as an input parameter for a specific tree growth model and can automatically estimate wood volume or stand stock. Compared with the LiDAR technology which is more suitable for large-scale operation, this paper can provide a more flexible method for the investigation of rare tree protection. The detailed attributes of precious trees need to be recorded during the operation. This paper presented a method which measures the properties of these precious trees and records the data in real time and stores it in the database of smartphones. In this paper, a scheme of "observation point transfer" is designed in the surveying work of plot level, but it takes a lot of space to describe the measurement of individual plant elevation. In terms of tree position measurement, mobile phone GNSS signal is greatly affected in some stands with high density of trees, resulting in low positioning accuracy. Also, the occluding GNSS signal of tree canopy is affected to varying degrees, and the absolute positioning technology based on GNSS signal may not be suitable for all stand positioning work $[28,30]$. The relative positioning technology of tree position in this paper makes up the problem that the smartphone-based GNSS absolute positioning technology cannot locate in the forest with weak signal. This method shows high accuracy, and it is less affected by terrain and tree density. Due to the difficulty of absolute positioning with smartphones in stand measurement, the relative positioning with smartphones is rarely studied to determine the position of trees. In the measurement of tree height and DBH of single tree, although some researchers have also used smartphones to measure tree height, most of the phones have simple principles and single functions with poor universality. Some researchers have developed smartphone software to measure tree height or DBH based on trigonometric principles $[5,7,9,41]$, but this method uses the phone's built-in angle sensor to get the angle of the observation point to the top and bottom of the tree. Because smartphone angle sensor sensitivity is different, the measurement error is unstable. In the actual work, because the sensor accuracy is insufficient, the result will have a large measurement error. In this paper, a high-precision sensor is attached to the smartphone to improve the measurement accuracy, and a single photo solution method of photogrammetry is applied to the monitoring of forest resources based on smartphone. Compared with other forest resource monitoring methods based on smartphones [26, 42-45], this method can directly measure the position, $\mathrm{DBH}$, and height 
of trees. The measurement accuracy will not be affected by different configurations of smartphones, so this method has the advantage of strong universality. Compared with the traditional method [46, 47], this method has computer power, does not need to touch trees, reduces the physical consumption of the surveyors, and saves the time and cost of the survey.

In order to give full play to the advantages of smartphones as microcomputers, this paper provides a low-cost and portable tool for current forest survey based on the method of measuring tree attributes based on smart space fusion of multiple sensors. Although surveyors can turn their smartphones into a professional tool for monitoring forest resources, there are still some problems that need to be further solved in this paper. The laser beam from the laser rangefinder module projects onto the tree trunk as a spot of light. When the light around the working environment is too weak or too strong, the visibility of the laser points in the tree trunk will be affected. In future research, this paper intends to change the geometry of the laser beam projected on the tree trunk from point to line. Finally, the laser beam is projected onto the tree trunk to form a laser line perpendicular to the tree trunk. Laser lines are more convenient for forest investigators than laser points. Compared with the way of setting up a smartphone by using a tripod in existing studies, the method in this paper is more convenient for the measurement personnel to carry and operate the smartphone, but it increases the instability of the attitude sensor in the sensor box to obtain the angle and direction data. During the collection process, the shaking of the arm will cause the measurement error, which will affect the accuracy of the tree position to some extent. In this paper, an image containing the complete information of standing trees is taken to calculate the $\mathrm{DBH}$ and tree height, which can complete the collection of sensor data in a very short time. The laser point is projected onto the tree trunk to minimize the error caused by the shaking of the arm. In this paper, on the premise of ensuring measurement accuracy, it is worthwhile to sacrifice less accuracy for the improvement of working efficiency and is more convenient to carry measurement tools. The sensor is still susceptible to electromagnetic interference, which reduces the measurement accuracy. The visibility of trees in forests remains the focus of further research in the future. Although we have designed an observation scheme of "observation point transfer" for the method in this paper, tree height cannot be directly measured for some totally closed stands. Therefore, the method of this paper is more suitable for small-scale, low-density, or medium-density forest resource monitoring with lower cost.

\section{Conclusions}

This paper presents a method of measuring key parameters of stand based on personal smart space fusion sensor. This paper designs a sensor box for personal smartphones that integrates various types of sensors and makes it easy to bind the two to each other. In order to verify the effectiveness of the method in this paper, the relative positioning algorithm and the standing tree image measurement algorithm can be used to measure the position, height, and $\mathrm{DBH}$ of the tree. And this software was developed to process the data collected by the sensor. Finally, the experimental results were analyzed and discussed. This paper has solved the problems of poor universality, low precision, and single function in the previous forest resource monitoring methods based on smartphones, so it has higher flexibility and better portability. Forest investigators can turn their smartphones into professional forest resource monitoring tools in the work field, which provides valuable help for forest resource monitoring. In the future, more forest resource monitoring factors, such as volume, crown width, and the height of living crown, need to be determined based on personal smart spaces. In general, this paper provides a new forest resource monitoring method. Based on personal intelligent space fusion of various high-precision sensors, this method can basically meet the precision requirements of forest resource monitoring and can be popularized in forestry survey.

\section{Data Availability}

The tree measurement data used to support the findings of this study are available from the corresponding author upon request.

\section{Conflicts of Interest}

The authors declare no conflicts of interest.

\section{Acknowledgments}

We thank other team members for help with the experiment. This research was jointly supported by the Fundamental Research Funds for the Central Universities (TD2014-02).

\section{References}

[1] S. M. Pawson, A. Brin, E. G. Brockerhoff et al., "Plantation forests, climate change and biodiversity," Biodiversity and Conservation, vol. 22, no. 5, pp. 1203-1227, 2013.

[2] Z. Qiu, Z. Feng, Y. Song, M. Li, and P. Zhang, "Carbon sequestration potential of forest vegetation in China from 2003 to 2050: predicting forest vegetation growth based on climate and the environment," Journal of Cleaner Production, vol. 252, Article ID 119715, 2020.

[3] M. Arnold, B. Powell, P. Shanley, and T. C. H. Sunderland, "EDITORIAL: forests, biodiversity and food security," International Forestry Review, vol. 13, no. 3, pp. 259-264, 2011.

[4] D. Han and C. Wang, "Tree height measurement based on image processing embedded in smart mobile phone," in Proceedings of the in 2011 International Conference on Multimedia Technology, pp. 3293-3296, Hangzhou, China, July 2011.

[5] X. Wu, S. Zhou, A. Xu, and B. Chen, "Passive measurement method of tree diameter at breast height using a smartphone," Computers and Electronics in Agriculture, vol. 163, Article ID 104875, 2019.

[6] A. Jaakkola, J. Hyyppä, A. Kukko et al., "A low-cost multisensoral mobile mapping system and its feasibility for tree measurements," ISPRS Journal of Photogrammetry and Remote Sensing, vol. 65, no. 6, pp. 514-522, 2010. 
[7] G. Fangli and X. U. Aijun, "Tree DBH measurement method based on smartphone and machine vision technology," Zjnldxxb, vol. 35, no. 5, pp. 892-899, 2018.

[8] D. Han, "Tree height measurement based on image processing with 3-points correction," in Proceedings of the 2011 International Conference on Computer Science and Network Technology, pp. 2281-2284, Harbin, China, December 2011.

[9] K. Zhou, Y. Wang, J. LI, G. Jiang, and A. Xu, "Research and implementation of tree measuring system based on Android platform," Journal of Nanjing Forestry University(Natural Sciences Edition), vol. 40, no. 4, pp. 95-100, 2016.

[10] A. Kangas, J. Rasinmäki, K. Eyvindson, and P. Chambers, "A mobile phone application for the collection of opinion data for forest planning purposes," Environmental Management, vol. 55, no. 4, pp. 961-971, 2015.

[11] M. Molinier, C. López-Sánchez, T. Toivanen et al., "Relasphone-mobile and participative in situ forest biomass measurements supporting satellite image mapping," Remote Sensing, vol. 8, no. 10, p. 869, 2016.

[12] S. Korpilo, T. Virtanen, and S. Lehvävirta, "Smartphone GPS tracking-Inexpensive and efficient data collection on recreational movement," Landscape and Urban Planning, vol. 157, pp. 608-617, 2017.

[13] M. Bakula, P. Przestrzelski, and R. Kazmierczak, "Reliable technology of centimeter GPS/GLONASS surveying in forest environments," IEEE Transactions on Geoscience and Remote Sensing, vol. 53, no. 2, pp. 1029-1038, 2015.

[14] J. Tomaštík, J. Tomaštík, Š. Saloň, and R. Piroh, "Horizontal accuracy and applicability of smartphone GNSS positioning in forests," Forestry, vol. 90, no. 2, pp. 187-198, 2017.

[15] G. Fan, F. Chen, Y. Li, B. Liu, and X. Fan, "development and testing of a new ground measurement tool to assist in forest GIS surveys," Forests, vol. 10, no. 8, p. 643, 2019.

[16] A. Jaakkola, J. Hyyppä, X. Yu et al., "Autonomous collection of forest field reference-the outlook and a first step with UAV laser scanning," Remote Sensing, vol. 9, no. 8, p. 785, 2017.

[17] T. Mikita, P. Janata, and P. Surový, "Forest stand inventory based on combined aerial and terrestrial close-range photogrammetry," Forests, vol. 7, no. 12, p. 165, 2016.

[18] L. Noordermeer, O. M. Bollandsås, H. O. Ørka, E. Næsset, and T. Gobakken, "Comparing the accuracies of forest attributes predicted from airborne laser scanning and digital aerial photogrammetry in operational forest inventories," Remote Sensing of Environment, vol. 226, no. 1, pp. 26-37, 2019.

[19] G. D. Pearse, J. P. Dash, H. J. Persson, and M. S. Watt, "Comparison of high-density LiDAR and satellite photogrammetry for forest inventory," ISPRS Journal of Photogrammetry and Remote Sensing, vol. 142, pp. 257-267, 2018.

[20] D. R. Miller, C. P. Quine, and W. Hadley, "An investigation of the potential of digital photogrammetry to provide measurements of forest characteristics and abiotic damage," Forest Ecology and Management, vol. 135, no. 1-3, pp. 279-288, 2000.

[21] K. Fankhauser, N. Strigul, and D. Gatziolis, "Augmentation of traditional forest inventory and airborne laser scanning with unmanned aerial systems and photogrammetry for forest monitoring," Remote Sensing, vol. 10, no. 10, p. 1562, 2018.

[22] B. Talbot, M. Pierzchała, and R. Astrup, "Applications of remote and proximal sensing for improved precision in forest operations," Croatian Journal of Forest Engineering: Journal for Theory and Application of Forestry Engineering, vol. 38, no. 2, pp. 327-336, 2017.

[23] T. Sankey, J. Donager, J. Mcvay, and J. B. Sankey, "UAV lidar and hyperspectral fusion for forest monitoring in the southwestern USA," Remote Sensing of Environment, vol. 195, pp. 30-43, 2017.

[24] S. Krause, T. G. M. Sanders, J.-P. Mund, and K. Greve, "UAVbased photogrammetric tree height measurement for intensive forest monitoring," Remote Sensing, vol. 11, no. 7, p. 758, 2019.

[25] L. Wallace, A. Lucieer, Z. Malenovský, D. Turner, and P. Vopěnka, "Assessment of forest structure using two UAV techniques: a comparison of airborne laser scanning and structure from motion (SfM) point clouds," Forests, vol. 7, no. 12 , p. $62,2016$.

[26] R. d. Grote, "Estimation of crown radii and crown projection area from stem size and tree position," Annals of Forest Science, vol. 60, no. 5, pp. 393-402, 2003.

[27] D. Alder and T. J. Synnott, Permanent Sample Plot Techniques for Mixed Tropical Forest, Oxford Forestry Institute, University of Oxford, Oxford, UK, 1992.

[28] D. L. Evans, R. W. Carraway, and G. T. Simmons, "Use of global positioning system (GPS) for forest plot location," Southern Journal of Applied Forestry, vol. 16, no. 2, pp. 67-70, 1992.

[29] P. Němec, "Comparison of modern forest inventory method with the common method for management of tropical rainforest in the Peruvian Amazon," Journal of Tropical Forest Science, vol. 27, no. 1, pp. 80-91, 2015.

[30] R. R. Kennedy, Use of Smartphone and GIS Technology for Sustainable Forestry in Eastern Ontario, Morriest Hall, Ottawa, Canada, 2012.

[31] J. Blackard, M. Finco, E. Helmer et al., "Mapping U.S. forest biomass using nationwide forest inventory data and moderate resolution information," Remote Sensing of Environment, vol. 112, no. 4, pp. 1658-1677, 2008.

[32] G. Chirici, R. E. McRoberts, S. Winter et al., "National forest inventory contributions to forest biodiversity monitoring," Forest Science, vol. 58, no. 3, pp. 257-268, 2012.

[33] A. Villasante and C. Fernandez, "Measurement errors in the use of smartphones as low-cost forestry hypsometers," Silva Fennica, vol. 48, no. 5, p. 11, 2014.

[34] E. M. Mikhail, J. Bethel, and J. C. McGlone, Introduction to Modern Photogrammetry, Wiley, Hoboken, NJ, USA, 2001.

[35] K. B. Atkinson, Close Range Photogrammetry and Machine Vision, Whittles, Dunbeath Mill, UK, 1996.

[36] D. Gaffrey, B. Sloboda, M. Fabrika, and Š. Šmelko, “Terrestrial single-photogrammetry for measuring standing trees, as applied in the Dobroc virgin forest," Journal of Forest Science-UZPI (Czech Reoublic), vol. 47, pp. 75-87, 2001.

[37] K. Kansanen, J. Vauhkonen, T. Lähivaara, and L. Mehtätalo, "Stand density estimators based on individual tree detection and stochastic geometry," Canadian Journal of Forest Research, vol. 46, no. 11, pp. 1359-1366, 2016.

[38] Z. Qiu, Z.-K. Feng, M. Wang, Z. Li, and C. Lu, "Application of UAV photogrammetric system for monitoring ancient tree communities in beijing," Forests, vol. 9, no. 12, p. 735, 2018.

[39] Z. Qiu, Z. Feng, J. Jiang, Y. Lin, and S. Xue, “Application of a continuous terrestrial photogrammetric measurement system for plot monitoring in the beijing songshan national nature reserve," Remote Sensing, vol. 10, no. 7, p. 1080, 2018.

[40] W. Xinmei, X. Aijun, and Y. Tingting, "Passive measurement method of tree height and crown diameter using a smartphone," IEEE Access, vol. 8, pp. 11669-11678, 2020.

[41] D. Han, "Standing tree volume measurement technology based on digital image processing," in Proceedings of the International Conference on Automatic Control and Artificial Intelligence (ACAI 2012), pp. 1922-1925, Xiamen, China, March 2012. 
[42] M. I. Marzulli, P. Raumonen, R. Greco, M. Persia, and P. Tartarino, "Estimating tree stem diameters and volume from smartphone photogrammetric point clouds," Forestry: An International Journal of Forest Research, vol. 93, no. 3, pp. 411-429, 2020.

[43] M. Vastaranta, E. Latorre, V. Luoma, N. Saarinen, M. Holopainen, and J. Hyyppä, "Evaluation of a smartphone app for forest sample plot measurements," Forests, vol. 6, no. 12, pp. 1179-1194, 2015.

[44] J. Tomaštík, Š. Saloň, D. Tunák, F. Chudy, and M. Kardo, "Tango in forests-an initial experience of the use of the new Google technology in connection with forest inventory tasks," Computers \& Electronics in Agriculture, vol. 141, pp. 109-117, 2017.

[45] E. González Latorre, "Evaluation of a mobilephone application for sample plot measurement in russia," Thesis, University of Helsink, Helsinki, Finland, 2015.

[46] D. R. Bower and W. W. Blocker, "Notes and observations: accuracy of bands and tape for measuring diameter increments," Journal of Forestry, vol. 64, no. 1, pp. 21-22, 1966.

[47] J.-M. Binot, D. Pothier, and J. Lebel, "Comparison of relative accuracy and time requirement between the caliper, the diameter tape and an electronic tree measuring fork," The Forestry Chronicle, vol. 71, no. 2, pp. 197-200, 1995. 